\title{
Correction: 3-deazaneplanocin A protects against cisplatin-induced renal tubular cell apoptosis and acute kidney injury by restoration of E-cadherin expression
}

\author{
Jun $\mathrm{Ni}^{1,2}$, Xiying Hou ${ }^{1}$, Xueqiao Wang ${ }^{3}$, Yinfeng Shi ${ }^{1}$, Liuqing $\mathrm{Xu}^{1}$, Xiaoqing Zheng ${ }^{3}$, Na Liu ${ }^{1}$, Andong $\mathrm{Qiu}^{3}$ and
} Shougang Zhuang ${ }^{1,4}$

\section{Correction to: Cell Death \& Disease https://doi.org/10.1038/s41419-019-1589-y published online 01 May 2019}

After publication of this article, the authors found some mistakes in the author information. The correct list of author information should be as following:

Jun $\mathrm{Ni}^{1,2}$, Xiying $\mathrm{Hou}^{1}$, Xueqiao Wang ${ }^{3}$, Yinfeng $\mathrm{Shi}^{1}$, Liuqing $\mathrm{Xu}^{1}$, Xiaoqing Zheng ${ }^{3}$, $\mathrm{Na} \mathrm{Liu}^{1}$, Andong $\mathrm{Qiu}^{3}$, Shougang Zhuang ${ }^{1,4}$

${ }^{1}$ Department of Nephrology, Shanghai East Hospital, Tongji University School of Medicine, Shanghai, China

${ }^{2}$ Shanghai Institute of Immunology, Department of Immunology and Microbiology, Shanghai Jiao Tong University School of Medicine, Shanghai, China

${ }^{3}$ School of Life Sciences and Technology, Tongji University, China

\begin{abstract}
${ }^{4}$ Department of Medicine, Rhode Island Hospital and Brown University School of Medicine, Providence, RI, USA

Correspondence: Shougang Zhuang (szhuang@lifespan. org)

${ }^{1}$ Department of Nephrology, Shanghai East Hospital, Tongji University School of Medicine, Shanghai, China

${ }^{4}$ Department of Medicine, Rhode Island Hospital and Brown University School of Medicine, Providence, RI, USA
\end{abstract}

This has been corrected in both the PDF and HTML versions of the Article.

Published online: 18 July 2019 